\title{
Extended Measurement of Cosmic-Ray Electron and Positron Spectrum from CALET on the ISS
}

\author{
Shoji Torii* \\ Research Institute for Science and Engineering, Waseda University, Tokyo 162-0044, Japan \\ Institute of Physics, Faculty of Engineering, Kanagawa University, Yokohama 221-8686, Japan \\ E-mail: torii.shojidwaseda.jp \\ Yoichi Asaoka \\ Research Institute for Science and Engineering, Waseda University,Tokyo 162-0044, Japan \\ E-mail: yoichi.asaokadaoni.waseda.jp

\section{for the CALET collaboration ${ }^{\dagger}$}

The CALET (CALorimetric Electron Telescope), launched to the International Space Station (ISS) on August 19, 2015 and accumulating scientific data since October 13, 2015, is an allcalorimetric instrument with total vertical thickness of 30 radiation lengths and fine imaging capability, optimized for a measurement of the electron and positron (all-electron) spectrum well into the TeV energy region. CALET will allow for the detailed search for various spectral structures of high-energy all-electron cosmic rays, perhaps providing the first experimental evidence of the presence of a nearby astrophysical cosmic-ray source.

All-electron spectrum analysis starts with detailed detector calibrations covering from detector alignment to energy determination, followed by electron event selection and flux normalization. Special care was taken to identify electrons in the presence of a large hadron background. Possible source of systematic errors are carefully surveyed, and associated uncertainties are estimated for each contribution. As a result, all-electron spectrum is obtained including the TeV energy range. In this contribution, we will present the all-electron spectrum measured by CALET and compare it with those obtained by other experiments.

36th International Cosmic Ray Conference -ICRC2019-

July 24th - August 1st, 2019

Madison, WI, U.S.A.

\footnotetext{
* Speaker.

${ }^{\dagger}$ for collaboration list see PoS(ICRC2019)1177
} 


\section{Introduction}

High-energy cosmic-ray electrons provide a unique probe of nearby cosmic accelerators. Electrons rapidly lose energy via inverse Compton scattering and synchrotron emission during propagation in the Galaxy. Since their diffusion distance above $1 \mathrm{TeV}$ is limited to less than $1 \mathrm{kpc}$, only a few super nova remnants as potential $\mathrm{TeV}$ sources are located in the vicinity of the Solar System. A precise measurement of the electron spectrum in the $\mathrm{TeV}$ region might reveal interesting spectral features to provide the first experimental evidence of the possible presence of a nearby cosmic-ray source [山, [2].

In addition, the apparent increase of the positron fraction over $10 \mathrm{GeV}$ established by Payload for Antimatter Matter Exploration and Light nuclei Astrophysics (PAMELA) [B] and the Alpha Magnetic Spectrometer (AMS-02) [䧃] may require a primary source component for positrons in addition to the generally accepted secondary origin. Candidates for such primary sources range from astrophysical (pulsar) to exotic (dark matter). Since these primary sources emit electronpositron pairs, it is expected that the all-electron (electrons and positrons) spectrum would exhibit a spectral feature, near the highest energy range of the primary component.

The CALET Collaboration managing the Calorimetric Electron Telescope (CALET) [[] , a space-based instrument optimized for the measurement of the all-electron spectrum, published its first result in the energy range from $10 \mathrm{GeV}$ to $3 \mathrm{TeV}$ [焑]. Subsequently, the Dark Matter Particle Explorer (DAMPE) Collaboration published their all-electron spectrum in the energy range from $25 \mathrm{GeV}$ to $4.6 \mathrm{TeV}[$ [ $]$.

In this paper, we present an extended CALET all-electron spectrum, using 780 days of flight data from October 13, 2015 to November 30, 2017 and the full geometrical acceptance in the highenergy region. We have increased our statistics by a factor of $\sim 2$ compared to Ref. [G]. The energy range is also extended up to $4.75 \mathrm{TeV}[\mathrm{B}]$. We will report at the conference a further study of the spectrum obtained by the observations over $\sim 3.5$ years.

\section{Instrument}

CALET employs a fully active calorimeter with 30 radiation-length thickness for particles at normal incidence. It consists of a charge detector (CHD), a 3 radiation-length thick imaging calorimeter (IMC), and a 27 radiation-length thick total absorption calorimeter (TASC), having a field of view of $\sim 45^{\circ}$ from zenith and a geometrical factor of $\sim 1,040 \mathrm{~cm}^{2} \mathrm{sr}$ for high-energy electrons. CHD, which identifies the charge of the incident particle, is comprised of a pair of plastic scintillator hodoscopes arranged in two orthogonal layers. IMC is a sampling calorimeter alternating thin layers of Tungsten absorber, optimized in thickness and position, with layers of scintillating fibers read-out individually. TASC is a tightly packed lead-tungstate (PbWO4; $\mathrm{PWO}$ ) hodoscope, capable of almost complete absorption of the TeV-electron showers.

Figure $\square$ shows a $3.05 \mathrm{TeV}$-electron candidate and a proton candidate with comparable energy deposit $(2.89 \mathrm{TeV})$ in the detector. Compared to hadron showers, which have significant leakage, the containment of the electromagnetic shower creates a difference in shower shape, especially in the bottom part of TASC, allowing for an accurate electron identification in the presence of a large hadron background. Together with the precision energy measurements from total absorption of 
electromagnetic showers, it is possible to derive the electron spectrum well into the $\mathrm{TeV}$ region with a straightforward and reliable analysis. The instrument was launched on August 19, 2015 and emplaced on the Japanese Experiment Module-Exposed Facility on the International Space Station with an expected mission duration of five years (or more). Scientific observations [8] were started on October 13, 2015, and smooth and continuous operations have taken place since then.
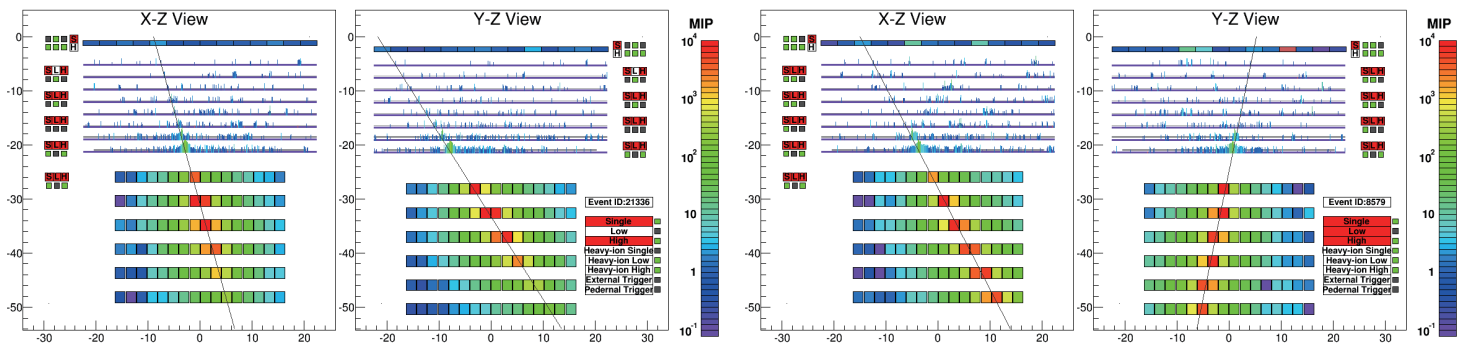

Figure 1: Examples of $\mathrm{TeV}$ event candidates showing energy deposit in each detector channel in the $\mathrm{X}$ $-\mathrm{Z}$ and $\mathrm{Y}-\mathrm{Z}$ views. (Left) An electron (or positron) candidate (reconstructed energy of $3.05 \mathrm{TeV}$ and energy deposit sum of $2.89 \mathrm{TeV}$ ). (Right) A proton candidate(energy deposit sum of $2.89 \mathrm{TeV}$ ).

\section{Data Analysis}

In this paper, we report the results obtained by analysis of 780 days of flight data collected with a high-energy shower trigger [ $[$ ] . The analysis was extended to use the full detector acceptance at higher energies as explained later, otherwise, it was done following the standard analysis procedure described in Ref. [焑].

A Monte Carlo (MC) program was used to simulate physics processes and detector response based on the simulation package EPICS [ए]] (EPICS9.20/COSMOS8.00). Using MC event samples of electrons and protons, event selection and event reconstruction efficiencies, energy correction factor, and background contamination were derived. An independent analysis based on GEANT4 [ㅁ] was performed, and small differences between the MC models are included in the systematic uncertainties. The GEANT4 simulation employs the hadronic interaction models FTFP_BERT as the physics list, while DPMJET3 [12] is chosen as the hadronic interaction model in the EPICS simulation.

While excellent energy resolution inside the $\mathrm{TeV}$ region is one of the most important features of a thick calorimeter instrument like CALET, calibration errors must be carefully assessed and taken into account in the estimation of the actual energy resolution. Our energy calibration [[13] includes the evaluation of the conversion factors between analog-to-digital converter units and energy deposits, ensuring linearity over each gain range (TASC has four gain ranges for each channel), and provides a seamless transition between neighboring gain ranges. Temporal gain variations occurring during long time observations are also corrected for in the calibration procedure [可]. The errors at each calibration step, such as the correction of position and temperature dependence, consistency between energy deposit peaks of non-interacting protons and helium, linear fit error of each gain range, and gain ratio measurements, as well as slope extrapolation, are included in the estimation of the energy resolution. As a result, a very high resolution of $2 \%$ or better is achieved above 20 $\mathrm{GeV}$ [123] . 
It should be noted that, even with such a detailed calibration, the determining factor for the energy resolution is the calibration uncertainty, as the intrinsic resolution of CALET is $\sim 1 \%$ as for DAMPE [14]. Intrinsic resolution refers to the detector's capability by design, taking advantage of the thick, fully active total absorption calorimeter. Also important is the fact that the calibration error in the lower gain ranges is crucial for the spectrum measurements in the $\mathrm{TeV}$ range.

We use the "electromagnetic shower tracking" algorithm [ㅍ] to reconstruct the shower axis of each event, taking advantage of the electromagnetic shower shape and IMC design concept. As input for the electron identification, well-reconstructed and well-contained single-charged events are preselected by (1) an off-line trigger confirmation, (2) a geometrical condition, (3) a track quality cut to ensure reconstruction accuracy, (4) a charge selection using CHD, (5) a longitudinal shower development, and (6) a lateral shower containment consistent with those expected for electromagnetic cascades.

In addition to fully contained events, the events incident from the IMC sides and exiting through the sides of TASC are used for analysis. For events not crossing the CHD, we use the energy deposit of the first hit IMC layer to determine their charge. The path length inside TASC is required to be longer than that of vertical depth of TASC, i.e. 27 r.l.

The energy of incident electrons is reconstructed using the energy correction function, which converts the energy deposit information of TASC and IMC into primary energy for each geometrical condition. In order to identify electrons and to study systematic uncertainties in the electron identification, we applied two methods: a simple two parameter cut and a multivariate analysis based on boosted decision trees (BDTs).

Calculation of event selection efficiencies, BDT training, and estimation of proton background contamination are carried out separately for each geometrical condition and combined in the end to obtain the final spectrum. Considering the fact that the lower energy region is dominated by systematics in our analysis, and therefore more statistics would not significantly improve the precision of our data, the fully-contained events are only included in the lower energy region below $475 \mathrm{GeV}$. Examples of a BDT response distribution including all acceptance conditions are shown in Fig. \. In the final electron sample, the resultant contamination ratios of protons are $\sim 5 \%$ up to $1 \mathrm{TeV}$, and $10 \%-20 \%$ in the $1-4.8 \mathrm{TeV}$ region, while keeping a constant high efficiency of $80 \%$ for electrons. The number of electron candidates in the highest energy bin is seven.
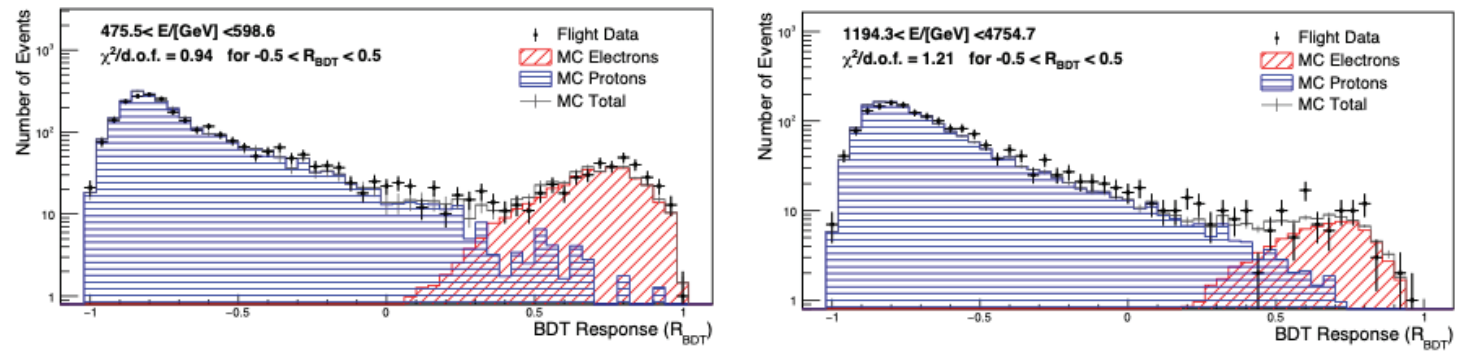

Figure 2: Examples of BDT response distributions in the $476<\mathrm{E}<599 \mathrm{GeV}$ bin (left) and in the highest region energy , $1196<\mathrm{E}<4755 \mathrm{GeV}$ bin. (left), including all acceptance conditions .

The absolute energy scale was calibrated and shifted by $+3.5 \%$ [目] as a result of a study of the geomagnetic cutoff energy. Since the full dynamic range calibration [ㅍ] was carried out with a scale-free method, its validity holds regardless of the absolute scale uncertainty. 


\section{Systematic Uncertainties}

Systematic uncertainties in our flux measurements can be divided into three categories, i.e., energy scale uncertainty, absolute normalization, and energy dependent uncertainties. As per the energy dependent systematics, we have identified the following contributions: trigger efficiency (below $30 \mathrm{GeV}$ ), BDT stability, tracking, charge identification, electron identification, and MC model dependence. BDT stability is evaluated from the stability of the resultant flux for 100 independent training samples and for BDT cut efficiency variation from $70 \%$ to $90 \%$ in $1 \%$ steps for each corresponding test sample.

Left and right panels of Fig. [3 show an example for the stability of the BDT analysis in the $949<\mathrm{E}<1194 \mathrm{GeV}$ bin and its energy dependence, respectively, where good stability over a wide range of efficiency factors and number of training samples is demonstrated. Dependence on tracking, charge identification, electron identification, and MC model is estimated by using the difference of the resultant flux between representative algorithms or methods, i.e., electromagnetic shower tracking vs combinatorial Kalman filter tracking [ए]] algorithms, CHD vs IMC charge identification methods, simple two parameter cut vs BDT cut, and the use of EPICS vs GEANT4, respectively.

The obtained energy dependence of the relative flux difference in each case is fitted with a suitable log-polynomial function to mitigate statistical fluctuations. Systematic effects up to a few percent are seen in the energy range below the TeV region. Statistical fluctuations are the most important limiting factor for estimating systematic errors in the $\mathrm{TeV}$ region.
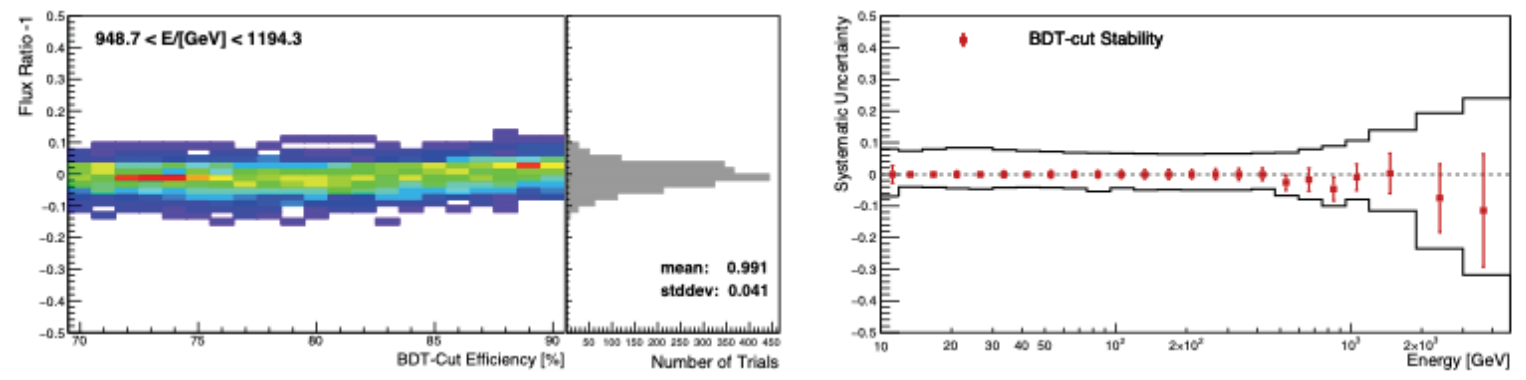

Figure 3: (Left) Stability of BDT analysis with respect to independent training samples and BDT-cut efficiency in the $949<\mathrm{E}<1194 \mathrm{GeV}$ bin. Color maps show the flux ratio dependence on efficiency, where the bin value (number of trials) increases as color changes from violet, blue, green, yellow to red. A projection onto the $\mathrm{Y}$-axis is shown as a rotated histogram (in gray color). (Right) Energy dependence of systematic uncertainties. The red squares represent the systematic uncertainties stemming from the electron identification based on BDT. The bands defined by black lines show the sum in quadrature of all the sources of systematics, except the energy scale uncertainties.

Since other selections, such as the track quality cut and shower concentration cuts, did not have a significant energy dependence, they were treated as uncertainties in the absolute normalization. Their contribution to the uncertainty in the absolute normalization was determined to be a very small part of the total. The total uncertainty in the absolute normalization was estimated to be $3.2 \%$. The high-energy trigger efficiency was verified by using data obtained with the low energy trigger ( $1 \mathrm{GeV}$ threshold) in the low rigidity cutoff region below $6 \mathrm{GV}$. By comparing the flux with and without off-line trigger confirmation, the systematic uncertainty from trigger efficiency 
is estimated to be $2.4 \%$ below $30 \mathrm{GeV}$, mainly limited by the available low-energy triggered data, and is negligible above this energy. The resultant flux for each of the acceptance conditions used in this analysis is consistent within the statistical uncertainty, indicating that there are no significant systematic deviations among the acceptance conditions.

\section{Electron + Positron Spectrum}

Figure $\mathbb{\theta}$ shows the extended electron and positron spectrum obtained with CALET [ $[8]$. The error bars along horizontal and vertical axes indicate bin width and statistical errors, respectively. The gray band is representative of the quadratic sum of statistical and systematic errors, using the same definition as the one given in Ref. [目]. Systematic errors include errors in the absolute normalization and energy dependent ones, except for the energy scale uncertainty. The energy dependent errors include those obtained from BDT stability, trigger efficiency in the low-energy region, tracking dependence, dependence on charge and electron identification methods, and MC model dependence. In more refined interpretation studies, the latter four contributions could be treated as nuisance parameters, while the first two components must be added in quadrature to the statistical errors. Conservatively, all of them are included in the total error estimate in Fig.

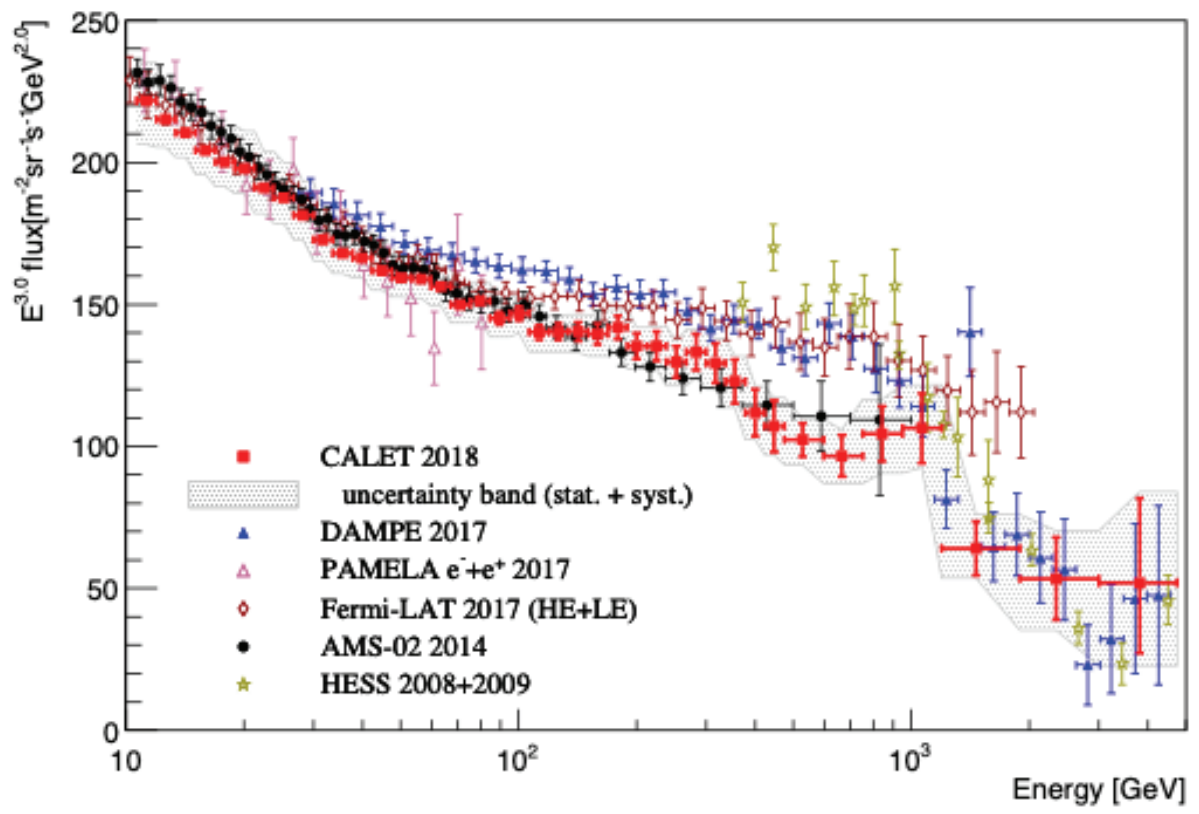

Figure 4: Cosmic-ray all-electron spectrum measured by CALET from $10.6 \mathrm{GeV}$ to $4.75 \mathrm{TeV}$ using the same energy binning as in our previous publication [目], where the gray band indicates the quadratic sum of statistical and systematic errors (not including the uncertainty on the energy scale). Also plotted are

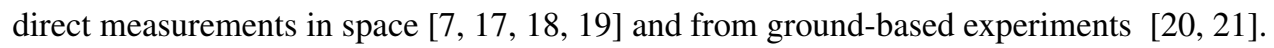

Comparing with other recent experiments [AMS-02, Fermi Large Area Telescope (FermiLAT), and DAMPE], our spectrum shows good agreement with AMS-02 data below $1 \mathrm{TeV}$. In the energy region from 40 to $300 \mathrm{GeV}$, the power-law index of CALET's spectrum is found to be $3.12 \pm 0.02$, which is consistent with other experiments within errors. However, the spectrum is considerably softer from 300 to $600 \mathrm{GeV}$ than the spectra measured by DAMPE and Fermi-LAT. 
The CALET results exhibit a lower flux than those of DAMPE and Fermi-LAT from $300 \mathrm{GeV}$ up to near $1 \mathrm{TeV}$, indicating the presence of unknown systematic effects.

To check if the CALET spectrum is consistent with a possible break at $0.9 \mathrm{TeV}$, as suggested by DAMPE's observations, we have adopted exactly the same energy binning as DAMPE to show our spectrum. In Fig. [], we fit our spectrum with a smoothly broken power-law model [ [0] in the energy range from $55 \mathrm{GeV}$ to $2.63 \mathrm{TeV}$, while fixing the break energy at $914 \mathrm{GeV}$. A broken power law steepening from $-3.15 \pm 0.02$ to $-3.81 \pm 0.32$ fits our data well, with $\chi^{2}=17.0$ and number of degrees of freedom (NDF) equal to 25; this result is consistent with DAMPE regarding the spectral index change of $0.7 \pm 0.3$. However, a single power-law fit over the same energy range gives an index $-3.17 \pm 0.02$ with $\chi^{2} / \mathrm{NDF}=26.5 / 26$, not a significantly poorer goodness of fit than obtained with the broken power law.

On the other hand, as presented in Fig. 6 , the flux in the 1.4 TeV bin of DAMPE's spectrum, which might imply a peak structure, is not compatible with CALET results at a level of $4 \sigma$ significance, including the systematic errors from both experiments.

Details of fitting of CALET's all-electron spectrum are described in Ref. [8] and the Supplement Material [22] with Table of the numerical values of the flux.

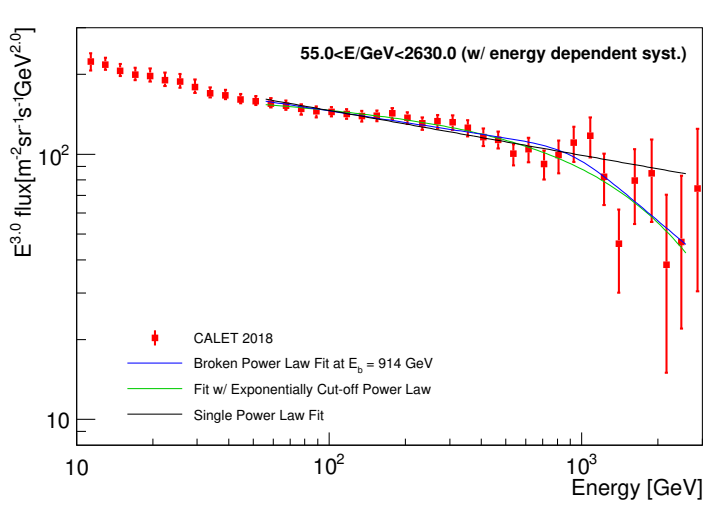

Figure 5: The fit of the CALET all-electron spectrum with a smoothly broken power law model presented by blue line, while fixing the break energy at $914 \mathrm{GeV}$ as determined by DAMPE [四] . A single power fit (black line) and an exponentially cut-off power law [23] (green line), are also presented for comparison.

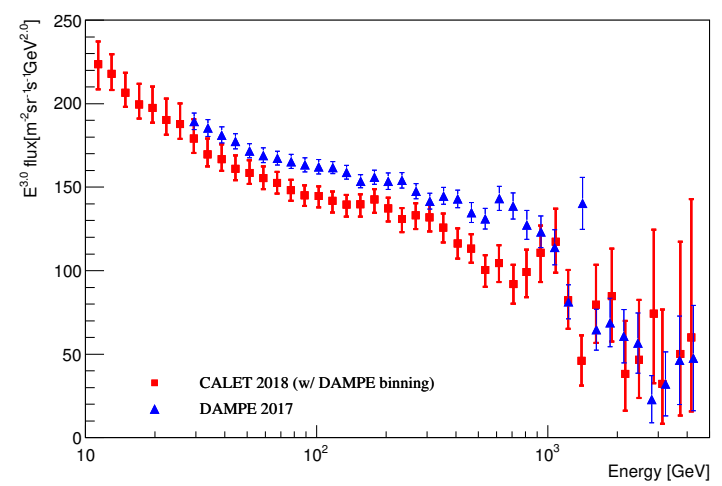

Figure 6: Cosmic-ray all-electron spectrum measured by CALET from $10.6 \mathrm{GeV}$ to $4.57 \mathrm{TeV}$ using the same energy binning as DAMPE's result [ $[0]$ and compared with it. The error bars indicate the quadratic sum of statistical and systematic errors (not including the uncertainty on the energy scale).

\section{Summary and Future Prospects}

In conclusion, we extended our previous result [G] on the CALET all-electron spectrum both in energy (to $4.8 \mathrm{TeV}$ ) and in acceptance, with an approximate increase by a factor of 2 of the statistics in the higher energy region. The data in the $\mathrm{TeV}$ region show a suppression of the flux compatible with the DAMPE results. However, the accuracy of the break's sharpness and position, and of the spectral shape above $1 \mathrm{TeV}$, will improve by better statistics and a further reduction of the systematic errors based on the analysis of additional flight data during the ongoing five-year (or more) observation. By specifying the breakdown of systematic uncertainties, our extended all-electron 
spectrum together with the AMS-02 positron flux measurement provides essential information to investigate spectral features in the framework of pulsars and/or dark matter inspired models.

\section{Acknowledgements}

We gratefully acknowledge JAXA's contributions to the development of CALET and to the operations onboard the International Space Station. We also wish to express our sincere gratitude to ASI and NASA for their support of the CALET project. This work was supported in part by a JSPS Grant-in-Aid for Scientific Research (S) (No. 26220708) and by the MEXT-Supported Program for the Strategic Research Foundation at Private Universities (No. S1101021) at Waseda University.

\section{References}

[1] J. Nishimura, et al., Astrophys. J. 238, 394 (1980).

[2] T. Kobayashi, Y.Komori, K.Yoshida, and J.Nishimura, Astrophys. J. 601, 340 (2004).

[3] O. Adriani, et al. , Nature (London) 458, 607 (2009).

[4] L. Accardo, et al., Phys. Rev. Lett. 113, 121101 (2014).

[5] S. Torii, et al., PoS(ICRC2017) 1092 (2018), https://pos.sissa.it/301/1092/pdf.

[6] O. Adriani, et al. , Phys. Rev. Lett. 119, 181101 (2017).

[7] G. Ambrosi, et al. , Nature (London) 552, 63 (2017).

[8] O. Adriani, et al. , Phys. Rev. Lett. 120, 261102 (2018).

[9] Y.Asaoka, Y.Ozawa, S.Torii et al., Atroparticle Physics 100, 29 (2018).

[10] K.Kasahara, EPICS, http://cosmos.n.kanagawa-u.ac.jp/EPICSHome/.

[11] S. Agostinelli, et al., Nucl. Instrum. Methods Phys. Res., Sect. A 506, 250 (2003).

[12] S. Roesler, R. Engel, and J. Ranft, DPMJET3, http://sroesler.web.cern.ch/sroesler/dpmjet3.html.

[13] Y. Asaoka, Y. Akaike, Y. Komiya, R. Miyata, S. Torii , et al. Astropart. Phys. 91, 1 (2017).

[14] J. Chang, et al., Astropart. Phys. 95, 6 (2017).

[15] Y. Akaike, etal., in Proceedings of 33rd International Cosmic Ray Conference (ICRC2013) 726 (2013), http://www.cbpf.br/ icrc2013/papers/icrc2013-0726.pdf.

[16] P. Maestro, M. Mori, et al., PoS(ICRC2017), 208, https://pos.sissa.it/301/208/pdf.

[17] O. Adriani, et al., Riv. Nuovo Cimento 40, 473 (2017).

[18] S. Abdollahi, et al., Phys. Rev. D 95, 082007 (2017).

[19] M. Aguilar, et al., Phys. Rev. Lett. 113, 221102 (2014).

[20] F. Aharonian, et al., Phys. Rev. Lett. 101, 261104 (2008).

[21] F. Aharonian, et al., Astron. Astrophys. 508, 561 (2009).

[22] O. Adriani, et al. , http://link.aps.org/ supplemental/10.1103/PhysRevLett.120.261102.

[23] S. Abdollahi, et al., Phys. Rev. D 95, 082007 (2017). 